\title{
Simulation based Behavioral Study of AODV, DSR, OLSR and TORA Routing Protocols in Manet
}

\author{
Pooja Singh \\ M.Tech(IT) \\ Banasthali University \\ Rajasthan India
}

\author{
Anup Bhola \\ Assistant Professor \\ Banasthali University \\ Rajasthan India
}

\author{
C.K Jha, PhD. \\ Associate Professor \\ Banasthali University \\ Rajasthan India
}

\begin{abstract}
The mobile Adhoc network is a concept of communication without planning in that mobile node communicate using different topology. The main agenda of this paper is how to receive the highest performance in wireless environments for this we evaluated some protocols like AODV, DSR, OLSR and TORA on OPNET 14.5 and analysis the performances of respective protocols on certain parameters like delay, load, media access delay, data dropped buffer-overflow, data dropped retry threshold exceeded and throughput under consider Banasthali network. Generally, Banasthali has wired network in many departments and hostels now we want to convert a total network of Banasthali into the wireless network.
\end{abstract}

\section{General Terms}

Adhoc, Manet, AODV, DSR, OLSR and TORA .

\section{Keywords}

Unit 14.5, Wlan, LAN, TC (Topology Control) and ATR ( Augmented Tree-based Routing).

\section{INTRODUCTION}

Banasthali is one of the five universities in India which offers an integrated system extending from the primary to the $\mathrm{PhD}$. level. When Banasthali campus network was established, it was small now continuous expanding size of the network makes our campus network very complex. Every year new department is open and complexity of the network is increasing. Now Banasthali University is having about 10,000 students, 1700 staffs, 28 departments and 31 hostels about 850 acres. The wireless network at the educational institution has started to be used to connect staff and students to outside corporate network. The entire University campus is networked through optical fiber LAN and powerful layer 3 and layer 2 (managed \& unmanaged) switches and Cisco 1700, 1800 and 2620 routers housed at the Apaji Institute. Banasthali uses both wired as well as wireless technology. It offers high efficiency of the network. As the university grows day by day Banasthali upgrades by wired LAN to wireless LAN because WLAN provides mobility. In modern communication systems, wireless Adhoc networking has become a unique technology where communication infrastructure is insufficient or unavailable. An Adhoc network is a collection of selforganizing nodes that are rapidly deployable and adaptable to frequent topology changes [1]. This paper represents a comparison of four routing protocols such as AODV, DSR, OLSR and TORA.
AODV (On-Demand Distance Vector Routing) means reactive distance vector based routing protocol, source node broadcasts a RREQ with parameters such as destination number, sequence number, hop-count etc. AODV uses simple metrics such as hop-count to perform routing, thereby providing with quick and easy solution. DSR (Dynamic Source Routing) uses a unique way of routing packets where the source of the packet has a list of nodes that the packet must go through to reach the destination. OLSR (Optimized Link State Routing Protocol) is a proactive link -state routing protocol, that uses hello and (TC) topology control messages to detect and then announce link state information throughout the mobile Adhoc network .TORA ( Temporally-ordered routing algorithm) is based on on-demand routing protocol. The main objective of TORA is to check control message propagation in the extremely dynamic mobile computing environment. Each node has to explicitly launch a query when it wants to send data to a specific destination. The main objective of this paper is how to obtain the highest performance in wireless environments for this we evaluated four routing protocols such as AODV, DSR, OLSR and TORA, and compare their performances on the basis of certain parameters under consider a network of Banasthali.

\begin{tabular}{|c|l|l|}
\hline S.N0 & Parameters & \multicolumn{1}{|c|}{ Description } \\
\hline 1. & $\begin{array}{l}\text { Daffer Overflow } \\
\text { (bits/sec) }\end{array}$ & $\begin{array}{l}\text { The total size of higher layer } \\
\text { data packets (in bits/sec) } \\
\text { dropped by all the WLAN } \\
\text { MACs in the network due to: } \\
\text { a) Full higher layer data buffer, } \\
\text { or }\end{array}$ \\
\hline 2. & $\begin{array}{l}\text { Data Dropped } \\
\text { (Retry } \\
\text { Threshold } \\
\text { Exceeded) } \\
\text { (bits/sec) }\end{array}$ & $\begin{array}{l}\text { The size of the higher layer } \\
\text { packet [2] } \\
\text { (in bits/sec) dropped by the all } \\
\text { the WLAN MACs in the } \\
\text { network as a result of } \\
\text { consistently failing } \\
\text { retransmissions[2]. }\end{array}$ \\
\hline 3. & Delay(sec) & $\begin{array}{l}\text { Represents the end to end delay } \\
\text { of all the packets received by } \\
\text { the wireless LAN MACs of all } \\
\text { WLAN nodes in the network } \\
\text { and forwarded to the higher } \\
\text { layer [2]. }\end{array}$ \\
\hline
\end{tabular}




\begin{tabular}{|c|l|l|}
\hline 4. & Load(bits/sec) & $\begin{array}{l}\text { Represents the total load (in } \\
\text { bits/sec) submitted to wireless } \\
\text { LAN layers by all higher layers } \\
\text { in al WLAN nodes of the } \\
\text { network[2]. }\end{array}$ \\
\hline 5. & $\begin{array}{l}\text { Media Access } \\
\text { delay (sec) }\end{array}$ & $\begin{array}{l}\text { For each frame, this delay is } \\
\text { calculated as the duration from } \\
\text { the time when it is inserted into } \\
\text { the transmission queue, which } \\
\text { is arrival time for higher layer } \\
\text { data packets and creation time } \\
\text { for all other frames types, until } \\
\text { the time when the frame is sent } \\
\text { to the physical layer for the } \\
\text { first time [2] }\end{array}$ \\
\hline 6. & $\begin{array}{l}\text { Throughput(bits/ } \\
\text { sec) }\end{array}$ & $\begin{array}{l}\text { Represents the total number of } \\
\text { bits (in bits/sec) forwarded } \\
\text { from wireless LAN layers to } \\
\text { higher layers in all WLAN } \\
\text { nodes of the network [2]. }\end{array}$ \\
\hline
\end{tabular}

All these parameters help us to evaluate the best routing protocol between them. All the parameters that have taken play a very vital role to judge or evaluate the performance of the wireless network. The target are to formulate observations practically how the performance of these protocols can change the performance of the Banasthali network .

\section{RELATED WORK}

In Adhoc networks scalability is a demanding needs if those mechanization has to extend their full latent. In July-2012 Pankaj Palta and Sonia Goyal [3] proposed a report, reactive routing protocols and Pro-reactive routing Protocols such as Optimized Linked State Routing (OLSR) and Temporally Ordered Routing Algorithm (TORA), are compared on the basis of a few parameters. In June 2012 Devendra Singh, Vandana Dubey and Shipra Sharma [4] discussed the relative study of the routing protocols such as AODV and DSR and also did a comparative study that which one is better with respect to different aspects. In 2012 Rajneesh Gujral, Sanjeev and Amrita Chaudhary analysis [5] an overview of major directions in previous researches on mesh and tree based multicast routing protocol in MANET. The primary focus is on applications and traffic management, rather than device attachment. They first review the pertinent aspects of network architecture and discuss the main difference between mesh and tree based multicast routing protocols [5]. In September 2011 Sandeep Kaur, Nitin Bhatia and Namarta Kapoor [6] proposed simulation analysis of the AODV Protocols consider two networks one having smaller nodes and other having larger nodes. In April 2011 Anil Kumar Sharma and Neha Bhatia [7] created a choice guide of routing protocol for network scenario. In 2010 Sunil Taneja and Ashwani Kush [8] introduce a paper name as A Survey of Routing Protocols in Mobile Ad Hoc Networks in this they provide an overview of DSR, AODV and TORA protocols by presenting their characteristics, functionality, benefits and limitations and then make their comparative analysis so to analyze their performance. In 2010 the paper name Based on MANET the Correctness and Efficiency Evaluation of DSR, AODV and TORA Routing Protocols for Best QOS, Jahangir khan [16] compared the performance of ad-hoc routing protocols like AODV, DSR and TORA in order to prove its correctness and efficiency evaluation of the protocols and to implement
AODV and DSR routing protocols by OPNET simulator to check the performance with respect to different parameters that mobility model changes with a significant impact on their performance and QoS support in different ways.

\section{SIMULATION ENVIRONMENT}

There are numbers of simulators available to perform the simulation of the design network. Basically simulator is used to evaluate the idea of planning about the network. OPNET MODULER 14.5 is one of the best simulator, used in this paper. For the experimental results first designed the small part of Banasthali network shown below.

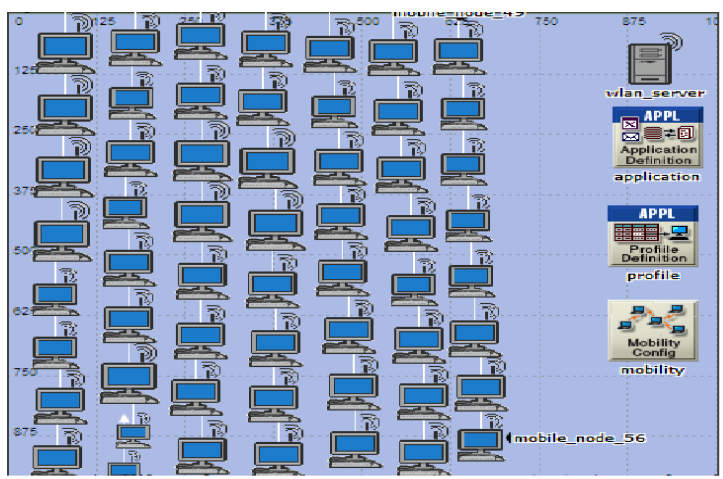

Fig 1. Scenario of 57 nodes

On above scenario we applied AODV, DSR OLSR and TORA routing protocols under some simulation parameters.

\begin{tabular}{|l|l|}
\hline Area of Experiment & Campus $\left(1000 * 1000 \mathrm{~m}^{2}\right)$ \\
\hline Number of Nodes & 57 \\
\hline Protocols & $\begin{array}{l}\text { AODV, DSR, OLSR and } \\
\text { TORA }\end{array}$ \\
\hline Performance Parameters & $\begin{array}{l}\text { Data Dropped Buffer } \\
\text { Overflow, Data Dropped } \\
\text { Retry Threshold Exceeded, } \\
\text { Delay, load, Media Access } \\
\text { Delay and Throughput. }\end{array}$ \\
\hline Duration of Simulation & \multicolumn{1}{|c|}{$160 \mathrm{sec}$} \\
\hline
\end{tabular}

\section{RESULTS}

Here analyzation of the performance of all four routing protocols i.e. AODV, DSR , OLSR and TORA under five parameters such as delay, load, data dropped buffer flow, data dropped retry threshold value exceeded and throughput on consider Banasthali networking through an Opnet Modeler14.5 simulator take place. The measurement unit for all five parameters are in sec or bits per sec. 


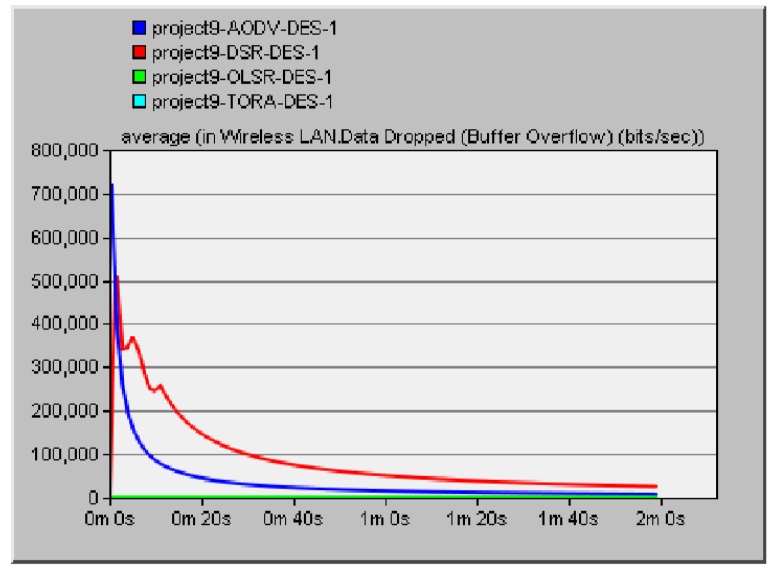

Fig 2.Data Dropped Bufferoverflow(bits/sec)

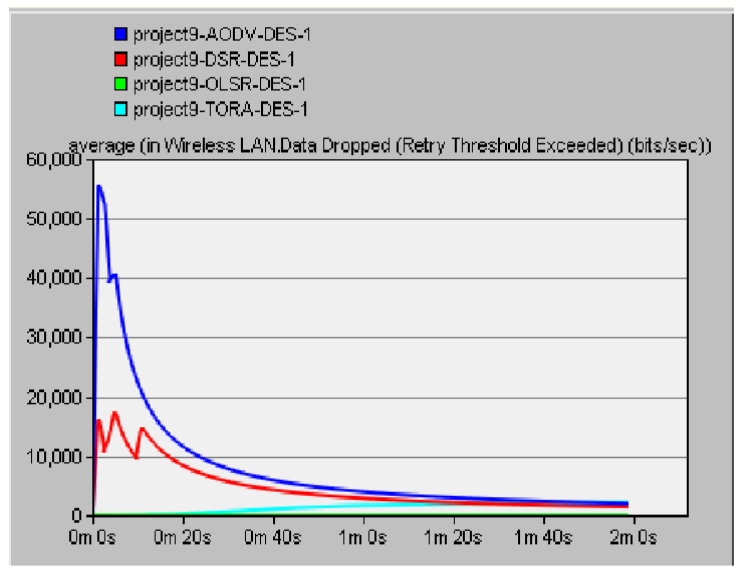

Fig 3. Data Dropped (Retry Threshold Exceeded(bits/sec)

Above graphs show the experimental results of two parameters i.e. data dropped buffer overflow and data dropped (Retry threshold Exceeded) under consider Banasthali network of 57 nodes. Both the axes of the graph represent time, $x$-axis represents simulation time and the $y$ axis represents the actual time taken by parameters. All routing protocols performed almost similar performance, among all four under both parameters OLSR routing protocol performed better shows almost zero sec data dropped .

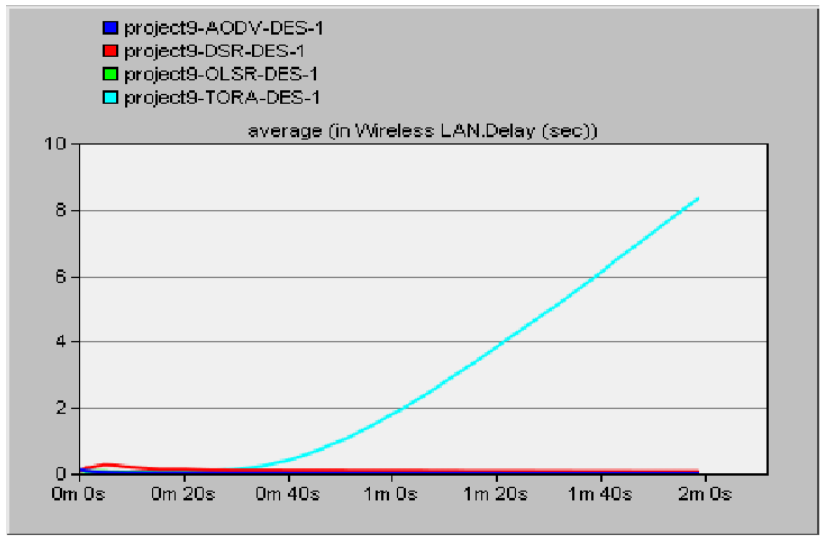

Fig 4. Delay

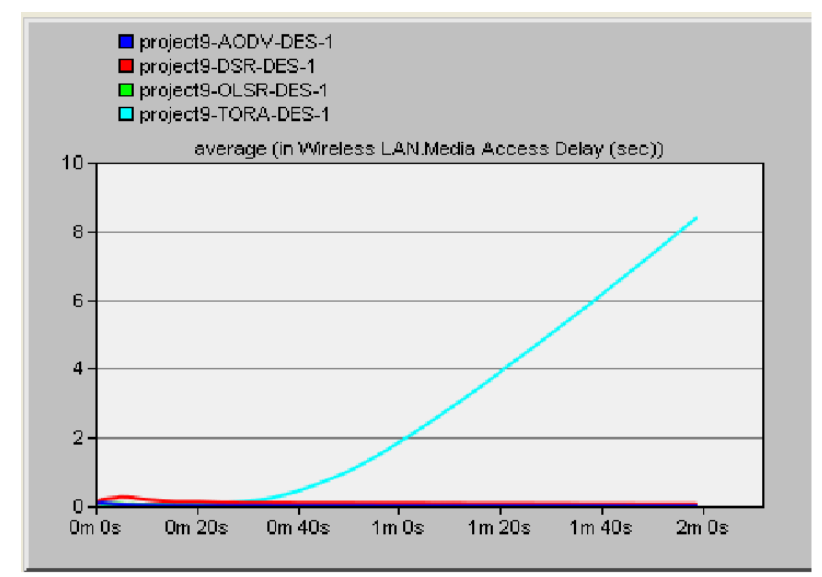

Fig 5.Media Access delay

Above graphs 4.3 and 4.4 show the performances of four routing protocols under delay and Media access delay in second. On the basis of graphs we conclude that an AODV routing protocol gives less delay and media access delay near about 0.011 and 0.013 secs respectively, but OLSR also able to give less delay nearly equal to 0.024 and 0.025 secs respectively as compare to all four routing protocols

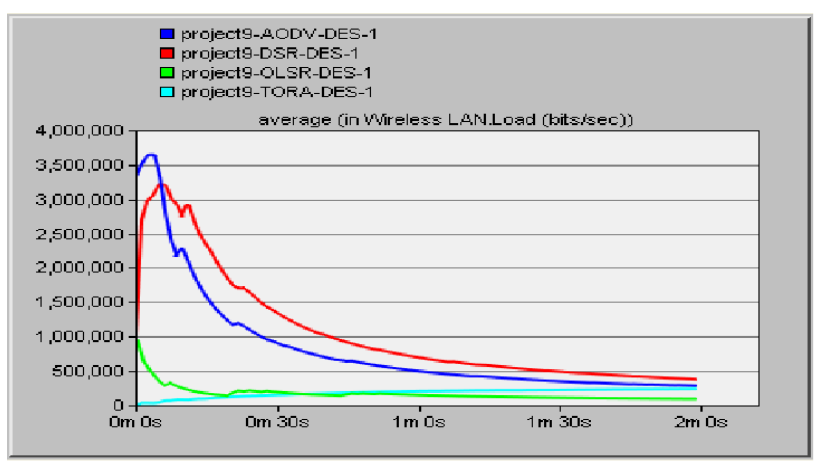

Fig 6. Load 


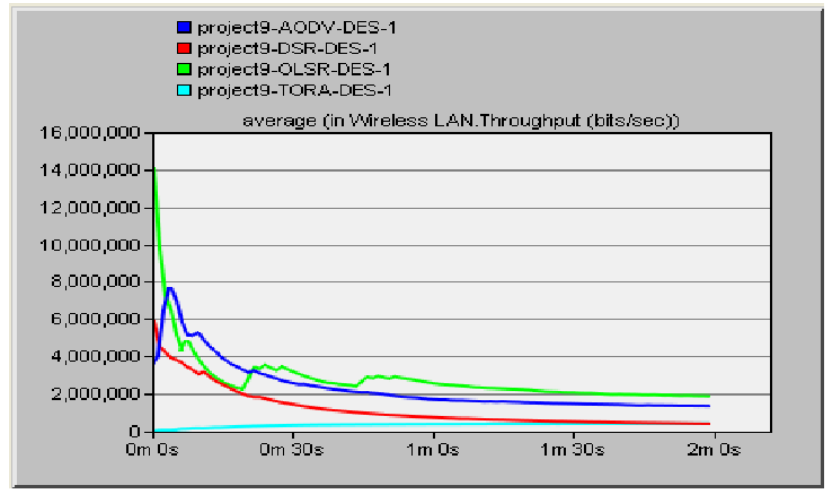

Fig 7. Throughput

Above graphs 4.5 and 4.6 represent the performances of all four routing protocols under load and throughput .In graph 4.5 OLSR routing protocol perform better as compared to each other in the total load (in bits/sec) submitted to wireless LAN layers by all higher layers in all WLAN nodes of the network nearly equal to $166,064.1169$. Fig 4.6 shows the throughput of all four routing protocols and OLSR routing protocol represents the higher throughput near equal to 2,836,395.614 secs among all four routing protocols.

\section{CONCLUSION}

This paper concludes that under consider a network of Banasthali different protocols gives almost similar performances. For example in particular parameters like delay and media access delay, AODV can perform better among all routing protocols but finally OLSR routing protocol gives an efficient performance under maximum parameters. On the basis simulations result, Banasthali electing OLSR routing protocol for Wireless network.

\section{ACKNOWLEDGMENTS}

I wish to thank the Dr.C.K Jha and Mr. Anup Bhola who gave their precious time, knowledge, experience for significant encouragement and support. I would also like to thank anonymous reviewers who gave valuable instructions that has comforted to enhance the quality of the paper. The practical intelligence in the field of OPNET those Lab Assistants gave are too valuable.

\section{REFERENCES}

[1] Jangeun Jun.2007."Networking in Wireless Ad Hoc Networks,"UMImicroform 327937.

[2] OPNET Technologies, Available at: http://www.opnet.com

[3] Pankaj Palta, Sonia Goyal.July 2012. "Comparison of OLSR and TORARouting Protocols Using OPNET Modeler," International Journal of Engineering Research \& Technology., vol. 1, Issue 5, pp: 2278-0181.
[4] Devendra Singh, Vandana Dubey, Shipra Sharma.June 2012" Performance Analysis of DSR and AODV in Manets: Using WLAN Parameters" International journal of computer Application Vol 47-No.3, pp: 0975-88.

[5] Rajneesh Gujral, Sanjeev Rana Amrita Chaudhary. July 2012"Study and Comparison of Mesh and Tree- Based Multicast Routing Protocols for MANETs", International Journal of Latest Trends in Engineering and Technology, Vol 1 Issue 2 pp: 2278-621X.

[6] Sandeep Kaur, Nitin Bhatia, Namarta Kapoor. September 2011" Simulation Analysis of AODV Routing Protocol of MANET using OPNET" International Journal of Computer Science and Technology, Vol 2, Issue 3, pp: 2229-4333 (Print) |pp: 0976-8491 (Online).

[7] Anil Kumar Sharma, Neha Bhatia. April 2011" International Journal of computational Engineering \& Management", Vol 12, pp (Online): 2230-7893.

[8] Sunil Taneja, Ashwani Kush. August 2010" A Survey of Routing Protocols in Mobile Ad Hoc Networks", International Journal of Innovation, Management and Technology, Vol 1 no 3, pp: 2010-0248.

[9] K. Prabhu, A. Subramani.September 2012'International Journal of Advanced Research in Computer Science and Software Engineering”, Vol 2, Issue 9, pp: 2277 128X.

[10] Khatri. P, Rajput. M, ShastriA, Solanki. K. 2010" Performance study of ad-hoc reactive protocols", journal of computer science, Vol 6 no 10 pp: 1130-1134.

[11] Nor Surayati Mohamad Usop, Azizol Abdullah. July 2009"Performance Evaluation of AODV, DSDV \& DSR Routing Protocol in Grid Environment", IJCSNS International Journal of Computer Science and Network Security, Vol 9 no 7 pp: 261.

[12] C.M barushimana, A. Shahrabi.May, 2003"Comparative study of Reactive and proactive Routing Protocols Perforamnce in Mobile Ad-Hoc Networks,"Workshop on Advance Information Networking and Application, Vol 2, pp: 679-684.

[13] Hetal Jasani, Kang Yen. June 2006" Performance Improvement using Directional Antennas in Ad Hoc"in proceeding of the IJCSNS International Journal of Computer Science and Network Security, VOL.6 No.

[14] Anuj K. Gupta, Member, IACSIT, Dr. Harsh Sadawarti, Dr. Anil K. Verma . April 2010 "Performance analysis of AODV, DSR\&TORA Routing Protocols" IACSIT International Journal of Engineering and Technology, Vol.2, No.2.

[15] Patrick Sondi, Dhavy Gantsou and Sylvain Lecomte March 2010."Performance Evaluation of Multimedia Applications over an OLSR-based Mobile Ad Hoc Network using OPNET," In the Proceedings of the $12^{\text {th }}$ International Conference on Computer Modelling and Simulation, Cambridge, UK,. pp. 562-572.

[16] Jahangir khan.2010" Based on MANET the Correctness and EfficiencyEvaluation of DSR, AODV and TORA Routing Protocols for Best QOS”, IJCA Special Issue on "Mobile Ad-hoc Networks. 